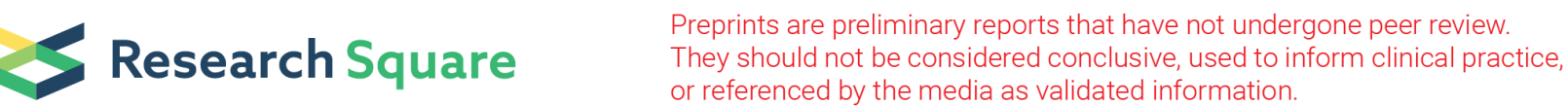

\section{Anti-SARS-CoV-2 Spike Protein and Anti-Platelet Factor 4 Antibody Responses Induced by COVID-19 Disease and ChAdOx1 nCov-19 vaccination}

Andreas Greinacher ( $\sim$ andreas.greinacher@med.uni-greifswald.de )

University Medicine Greifswald

Kathleen Selleng

University Medicine Greifswald

Julia Mayerle

German Centre for Infection Research (DZIF)

Raghavendra Palankar

University Medicine Greifswald

Jan Wesche

University Medicine Greifswald

Sven Reiche

Friedrich-Loeffler Institut, Greifswald-Insel Riems

Andrea Aebischer

Friedrich-Loeffler Institut, Greifswald-Insel Riems

Theodore E. Warkentin

McMaster University

Maximilian Muenchhoff

Ludwig Maximilians University of Munich

Johannes C. Hellmuth

University Hospital Munich

Oliver T. Keppler

Ludwig Maximilians University of Munich

Daniel Duerschmied

University of Freiburg

Achim Lother

University of Freiburg

Siegbert Rieg

University of Freiburg

Meinrad Paul Gawaz

University Tuebingen

Karin Anne Lydia Mueller 
University Tuebingen

\section{Christian S. Scheer}

University Medicine Greifswald

\section{Matthias Napp}

University Medicine Greifswald

\section{Klaus Hahnenkamp}

University Medicine Greifswald

\section{Guglielmo Lucchese}

University Medicine Greifswald

\section{Antje Vogelgesang}

University Medicine Greifswald

\section{Agnes Flöel}

University Medicine Greifswald

\section{Piero Lovreglio}

University of Bari

\section{Angela Stufano}

University of Bari

\section{Rolf Marschalek}

Goethe University

\section{Thomas Thiele}

University Medicine Greifswald

\section{Research Article}

Keywords: Covid-19, PF4, VITT, spike protein vaccine

Posted Date: April 9th, 2021

DOl: https://doi.org/10.21203/rs.3.rs-404769/v1

License: (c) (7) This work is licensed under a Creative Commons Attribution 4.0 International License. Read Full License

Version of Record: A version of this preprint was published at Blood on July 19th, 2021. See the published version at https://doi.org/10.1182/blood.2021012938.

\section{EDITORIAL NOTE:}


This study explores a potential mechanism for the rare thrombotic events observed following vaccination with AstraZeneca's COVID-19 vaccine (AZD1222). The researchers conclude that antibodies against SARS-CoV-2 spike protein do not cross-react with platelet factor 4 , the protein targeted in this rare disorder. At the time of this posting, the World Health Organization has stated that "a causal relationship between the vaccine and the occurrence of blood clots with low platelets is considered plausible but is not confirmed." Some of the authors disclose relevant conflicts of interest. 


\section{Abstract}

Background: Some recipients of ChAdOx1 nCoV-19 COVID-19 Vaccine AstraZeneca develop antibodymediated vaccine-induced thrombotic thrombocytopenia (VITT), associated with cerebral venous and other unusual thrombosis resembling autoimmune heparin-induced thrombocytopenia. A prothrombotic predisposition is also observed in Covid-19. We explored whether antibodies against the SARS-CoV-2 spike protein induced by Covid-19 cross-react with platelet factor 4 (PF4/CXLC4), the protein targeted in both VITT and autoimmune heparin-induced thrombocytopenia.

Methods: Immunogenic epitopes of PF4 and SARS-CoV-2 spike protein were compared via prediction tools and 3D modelling software (IMED, SIM, MacMYPOL). Sera from 222 PCR-confirmed Covid-19 patients from five European centers were tested by PF4/heparin ELISA, heparin-dependent and PF4dependent platelet activation assays. Immunogenic reactivity of purified anti-PF4 and anti-PF4/heparin antibodies from patients with VITT were tested against recombinant SARS-CoV-2 spike protein.

Results: Three motifs within the spike protein sequence share a potential immunogenic epitope with PF4. Nineteen of 222 (8.6\%) Covid-19 patient sera tested positive in the IgG-specific PF4/heparin ELISA, none of which showed platelet activation in the heparin-dependent activation assay, including $10(4.5 \%)$ of the 222 Covid-19 patients who developed thromboembolic complications. Purified anti-PF4 and antiPF4/heparin antibodies from two VITT patients did not show cross-reactivity to recombinant SARS-CoV-2 spike protein.

Conclusions: The antibody responses to PF4 in SARS-CoV-2 infection and after vaccination with COVID19 Vaccine AstraZeneca differ. Antibodies against SARS-CoV-2 spike protein do not cross-react with PF4 or PF4/heparin complexes through molecular mimicry. These findings make it very unlikely that the intended vaccine-induced immune response against SARS-CoV-2 spike protein would itself induce VITT.

\section{Introduction}

Coronavirus disease 2019 (Covid-19) is caused by severe acute respiratory syndrome coronavirus 2 (SARS-CoV-2), a single-stranded RNA virus, encoding 16 non-structural proteins (NSP's 1-16), 8 accessory proteins (ORF3a, 6, 7a, 7b, 8, 9b, 9c and 10) and 4 structural proteins, known as S (spike), E (envelope), M (membrane), and $\mathrm{N}$ (nucleocapsid) proteins. ${ }^{1}$ The spike glycoprotein is responsible both for recognition of host cell membrane receptors, ACE2, and TMPRSS2 for mediating fusion with the host cell membrane. $^{2}$

Between December 2020 and March 2021, the European Medical Agency approved four vaccines ${ }^{3}$ for prevention of symptomatic Covid-19: two mRNA-based vaccines encoding the spike protein antigen of SARS-CoV-2, encapsulated in lipid nanoparticles, Comirnaty (BioNTech/Pfizer) and COVID-19 Vaccine Moderna; a recombinant chimpanzee adenoviral (ChAdOx1-S) vector encoding the spike glycoprotein of SARS-CoV-2, COVID-19 Vaccine AstraZeneca (Vaxzevria); and a recombinant adenovirus type 26 vector 
encoding SARS-CoV-2 spike glycoprotein, Covid-19 Vaccine Janssen. ${ }^{4,5}$ Thromboembolic complications in Covid-19 disease have been reported frequently, including unusual locations such as cerebral venous sinus thrombosis (CVST), even in patients without severe respiratory disease. ${ }^{6-8}$

Very recently, several cases of unusual thromboses such as CVST and splanchnic vein thrombosis in combination with moderate to severe thrombocytopenia were observed in healthy individuals approximately 5 to 20 days following vaccination with COVID-19 Vaccine AstraZeneca. ${ }^{9}$ Known as vaccine-induced thrombotic thrombocytopenia (VITT), we have identified immunoglobulin G class platelet-activating antibodies directed against the cationic platelet chemokine, platelet factor 4 (PF4; CXCL4), as the underlying cause. ${ }^{9}$ In such patients, platelet activation occurs via platelet Fcylla receptors. Patients often show laboratory signs of disseminated intravascular coagulation with severe thrombocytopenia without preceding heparin exposure. These features mimic the severe prothrombotic disorder, autoimmune heparin-induced thrombocytopenia (aHIT), which unlike classic HIT features heparin-independent platelet-activating antibodies. ${ }^{10,11}$

Some of these clinical features of thrombocytopenia and disseminated intravascular coagulation are also observed in Covid-19 patients. In addition, two recent studies showed Fcylla receptor-dependent platelet activation by sera of some Covid-19 patients. ${ }^{12,13}$ Further, patients with Covid-19 antibodies reacting strongly in the PF4/heparin ELISA have also been described, but these sera did not activate platelets in the presence of heparin. ${ }^{14}$ These antibodies were considered likely to represent an epiphenomenon attributed to the strong systemic inflammatory response of Covid-19. However, given that unusual thromboses such as CVST have been observed in Covid-19 as well as rarely in otherwise healthy individuals receiving the COVID-19 Vaccine AstraZeneca, the question arises as to whether the immune response against the spike protein could induce antibodies that cross-react with immunogenic antigens shared between spike protein and PF4. Accordingly, we sought to identify structural similarities between the SARS-CoV-2 spike protein and PF4. Further, we assessed binding characteristics of anti-PF4 antibodies obtained from patients with the newly recognized disorder, VITT, who developed unusual thrombotic complications. We compared their pattern of PF4-dependent platelet activation with those of sera obtained from a large series of Covid-19 patients from different independent cohorts. Our overall aim was to differentiate whether the reactivity patterns of antibodies in these two patient cohorts, Covid-19 patients immunized by the virus, and patients with VITT, indicate cross-reactivity between SARS-CoV-2 spike protein and PF4, or whether they are distinct entities.

\section{Material And Methods}

\section{Covid-19 Patient Cohorts}

A total of 222 Covid-19 patients were enrolled from five prospective registries from university medical centers in Munich (CORKUM, WHO trial ID DRKS00021225), Freiburg (WHO trial ID DRKS00021206), Tuebingen (approval by the local ethics committee 240/2018BO2), Greifswald (DRKS-ID: DRKS00023770) and Brescia, Italy (approval by the local ethics committee, ID Number: NP 4463). 
Patients between the age of 4 months to 88 years with available serum and positive PCR testing of SARSCoV-2 in nasopharyngeal swabs were enrolled. Registries began recruiting patients at varying start dates ranging from February 2020 until October 2020. Patient characteristics are summarized in Table 1; registries are described in detail in the Supplementary Material 1.

Sera from 6 VITT patients presenting with thrombocytopenia and thromboembolic events approximately 5-20 days after COVID-19 Vaccine AstraZeneca vaccination were available. ${ }^{9}$

\section{Identification of immunogenic epitopes and homologies of human PF4 and SARS-CoV-2 spike protein and the comparative analysis of their 3D structures}

The protein sequence for human PF4/CXCL4 was retrieved from the ENSEMBL gene data base (ENSG00000163737). ${ }^{15}$ Similarly, the protein sequence of the SARS-CoV-2 spike protein (1273 amino acids) was retrieved from publicly available data bases (NCBI: Gene ID 43740568)..$^{16}$ Using the online prediction tool of the University of Madrid, Spain (http://imed.med.ucm.es/Tools/antigenic.pl), ${ }^{17}$ we identified potential immunogenic peptide sequences (epitopes) in both protein sequences. In the SIM Alignment online Tool (https://web.expasy.org/sim/), ${ }^{18}$ the following default setting parameters were applied (comparison matrix BLOSUM62, gap opening penalty=12 and gap extension penalty=4). For 3D analysis, the MacMYPOL program (https://pymol.org/2/) ${ }^{19}$ was used together with the files $6 \mathrm{vxx} . \mathrm{pbd}$ and 4r9w.pbd available for the PBD database (http://www.rcsb.org) ${ }^{20}$ to compare the epitopes on the published structures of the proteins.

\section{Cloning and expression of SARS-CoV-2 spike protein}

The SARS-CoV2 spike ectodomain amino acids 17-1213 and the RBD-SD1 domain aa 319-519 (based on QHD43416) ${ }^{21}$ were cloned and expressed in the human cell line Expi293 (Thermo Fisher Scientific, Germany) (details Supplementary Material 2).

\section{Testing for PF4/heparin-reactive and platelet-activating immunoglobulin $\mathbf{G}$ antibodies}

For screening of all sera of the Covid-19 cohorts and the patients with VITT, we used an IgG-specific antiPF4/heparin ELISA, with antibody binding measured using a secondary antihuman IgG antibody, as described. ${ }^{22}$ Optical density (OD) results $<0.5$ units were considered negative, $\geq 0.5<1.0$ weak-positive, and $O D \geq 1.0$ strong-positive.

We performed platelet activation assays using purified, washed platelets from healthy volunteers, as described, ${ }^{9}$ using patient sera, or the respective purified anti-PF4/heparin IgG fractions with and without addition of PF4 (10 $\mu \mathrm{g} / \mathrm{mL})$ (Chromatec, Greifswald, Germany). Unfractionated heparin (100 IU/mL, final) was added to evaluate inhibition of antibody- and PF4-dependent platelet activation. Platelet activation was judged positive if at least two of 3 donor cells aggregated within 30 minutes. ${ }^{23,24}$

\section{Affinity purification of PF4 and PF4/heparin IgG antibodies}


Biotinylated PF4 (biotin-PF4) (Chromatec, Greifswald, Germany) and biotin-PF4/heparin complexes were coupled to streptavidin-conjugated paramagnetic microbeads (Dynabeads MyOne Streptavidin T1, Invitrogen). Beads were incubated with the serum, unbound antibodies and plasma removed by washing, and the IgG fractions were eluted (details in Supplementary Material 2).

\section{Binding studies of affinity purified anti-PF4 and anti-PF4/heparin IgG to SARS-CoV-2 S-1 domain, receptor-binding domain, full-length spike protein, PF4 and PF4/heparin complexes}

We identified sera testing positive for anti-PF4/heparin antibodies from two patient groups, (a) patients with Covid-19 disease (only a minority tested positive), and (b) patients with VITT (all tested positive). These sera were assessed for anti-spike protein antibodies using the SARS-CoV-2 full-length spike protein, the receptor-binding domain (RDB) using in-house ELISAs, and a commercially-available CoV-2 ELISA (recombinant S1-domain; El 2606-9620 G; EUROIMMUN Medizinische Labordiagnostika AG, Lübeck, Germany). Anti-PF4 and anti-PF4/heparin affinity-purified IgG fractions of two VITT patients with documented thromboembolic events were used in a 1:20 dilution (detailed description in Supplementary Material 2).

\section{Results}

\section{Identification of immunogenic peptide sequences (epitopes) in human PF4 and the SARS-CoV-2 spike protein}

A total of three and 63 potential immunogenic epitopes, respectively, were identified within the 70 amino acid sequence of PF4 (6-21, 23-43 and 49-66) and the 1273 amino acid long sequence of the SARS-CoV-2 spike protein (bold letters in the amino acid sequences, Supplementary Table S1A). The spike protein variants $\Delta H 69 \Delta V 70, E 484 K$ and N501Y (present in B.1.1.7, B.1.351, and P.1, respectively) showed the same immunogenic profile (data not shown).

Both proteins showed sequence homologies between 23.5\% and 66.7\% (Supplementary Table S1B). Overlapping homologous sequences varied between 5 and 22 residues in length. In addition, sequence identities were manually investigated for identical amino acids that are spaced by 2-3 amino acids to be localized on the same side of a particular motif. Restricting the search to motifs longer than 10 amino acids identified three motifs within the spike protein sequence (145-155, 323-335, and 677-694) that shared a potential immunogenic epitope with PF4. One of them is located in the structure file (6vxx.pbd: 323-335) and displays high similarity to two consecutive epitopes within PF4 (6-21/23-43).

For 3D comparison analysis, we used the following pbd-files: $6 \mathrm{vxx}$.pdf for the trimeric spike protein and 4r9w.pbd for dimeric PF4 bound to fondaparinux. Both the "15-27"- and the "323-335"-sequences display a ß-sheet-flexible loop structure. While the spike epitope resembles a planar configuration, the PF4 structure is more of a pleated sheet (see Figure 1A). Of interest, the same motif in PF4 is involved in binding fondaparinux and heparin (see Figure 1B). ${ }^{25}$ In Figure 1C, the surface epitope "323-335" is shown in one subunit of the trimeric SARS-CoV-2 spike protein (left), and the identified epitope is enlarged again 
in the magnified inset on the right. We assume that part of this epitope is similar in structure and shape surrounding the central Valine-Arginine motif between the spike protein and PF4. Binding of an antibody to this epitope may induce small conformational changes in PF4, similar to what has been observed by heparin binding to PF4.

\section{Covid-19 patient cohorts}

From the five patient cohorts, a total of 222 patients ( 125 males, 97 females; median age, 55 years [range, 4 months to 88 years]) were evaluated in the IgG-specific PF4/heparin ELISA. Nineteen of $222(8.6 \%)$ patients tested positive (above the 0.500 optical density threshold), with 13 testing in a range between OD 0.500 and $<1.000$ and 6 testing between OD 1.000 to <2.000) (Table 1). We did not observe differences in reactivity among the five different patient cohorts when analyzed per participating center, excluding preanalytical problems or a batch effect (data not shown).

Sera from all 19 patients who tested positive in the anti-PF4/heparin ELISA were tested in the platelet activation assay in the presence of heparin and of PF4, respectively, to judge heparin- and PF4-dependent platelet activation. Under reaction conditions previously shown to result in typically strong serum-induced platelet activation of COVID-19 Vaccine AstraZeneca vaccinated VITT patients (PF4, $10 \mu \mathrm{g} / \mathrm{mL}$ ), we found that 4/19 sera showed weak to moderate platelet activation in the presence of PF4 (lag time, median 15 minutes, range 10 to $>30 \mathrm{~min}$ [for non-reacting platelets], cut off $30 \mathrm{~min}$ ); in contrast, none of these sera showed platelet activation in the presence of 0.2 anti-factor $\mathrm{Xa} \mathrm{U} / \mathrm{mL}$ low-molecular-weight heparin. For 10/222 patients, thromboembolic complications were reported (six patients with pulmonary embolism, one patient with stroke, two patients with portal vein thrombosis, one thrombosis of unknown localization). Nine of these 10 patients tested negative by PF4/heparin ELISA. Only one serum was reactive with $O D>1.0$; for this patient a pulmonary embolism was reported. None of these ten sera, including from the patient with pulmonary embolism, induced platelet aggregation in the functional test, regardless of whether heparin or PF4 was added.

\section{No serological cross-reactivity of purified anti-PF4 and anti-PF4/heparin antibodies from VITT patient serum with recombinant SARS-CoV-2 spike protein}

As expected with an early primary immune response, the sera of (recently-vaccinated) VITT patients contained weakly to moderately binding IgG to the S1 sequence and the RBD sequence of the spike protein, with somewhat higher levels of optical density (OD) values to the full-length spike protein. In contrast, all VITT sera showed very strong binding to PF4 and PF4/heparin complexes (OD>3.0; Figure 2). Antibodies affinity purified using PF4 or PF4/heparin from two VITT sera also reacted strongly in the PF4 and PF4/heparin ELISA and strongly activated platelets in the presence of PF4 (data not shown), but did not bind to any of the SARS-CoV-2 spike protein constructs.

\section{Discussion}


The mechanism by which ChAdOx1 nCov-19 vaccination rarely induces antibodies that cause marked PF4-dependent platelet activation with resulting thrombocytopenia and unusual thromboses is unresolved. One potential mechanism is a general response of the immune system triggered either by vaccination or by the proinflammatory state of severe acute Covid-19. Clinical observations show unusually strong proinflammatory symptoms in the majority of individuals starting about eight to twelve hours post-vaccination, lasting for 12-24 hours. Potentially, this inflammatory response in certain individuals (e.g. by differences in their genetics, HLA type, or proinflammatory conditions) may have led to the observed occurrences of severe VITT ( $n=31$ patients reported in Germany at the time of writing). Reducing the vaccine dose of the COVID-19 Vaccine AstraZeneca might reduce inflammatory reactions. The published report from the first phase I/III study where a reduced dose of $2.5 \times 10^{10}$ viral particles was administered in the first vaccination shot indicates no marked difference in antibody response compared to the currently used dose. 26

Another possibility is that the immune response induced by infection with SARS-CoV-2 results in antibodies against the spike protein that also cross-react with PF4. In this scenario, Covid-19 vaccination could potentially trigger formation of especially strong anti-spike protein antibodies, cross-reacting with PF4 and thereby becoming highly pathogenic through anti-PF4-mediated platelet activation. Indeed, structural analysis of both the spike protein and PF4 indicated potential cross-reactive epitopes. However, by using purified recombinant spike protein, purified PF4, and affinity purified anti-PF4 antibodies from sera of VITT patients, we found no evidence for cross-reactivity. The platelet-activating anti-PF4 antibodies obtained from individuals with VITT post-vaccination with COVID-19 Vaccine AstraZeneca, did not cross-react with the spike protein on SARS-CoV-2. Of particular interest are the different magnitudes of antibody response against both proteins, indicating two different immune responses. ${ }^{9}$ The VITT patients showed strong antibody reactivity against PF4 within 5-14 days post-vaccination presumably reflecting a secondary immune response. A primary immune response is extremely unlikely to yield such high IgG reactivity (titers $>1: 3,000$; data not shown). Precedence for this concept is found in the heparininduced thrombocytopenia literature: patients who develop this complication with their first heparin exposure develop a strong IgG immune response beginning as early as 4-5 days post-immunizing heparin exposure ${ }^{27,28}$ consistent with prior presensitization through naturally-occurring polyanions. ${ }^{29-31}$

When we performed a combined analysis of five patient cohorts comprising 222 Covid-19 patients with variable clinical disease severity, we found no evidence for an association between anti-PF4/heparin IgG and thromboembolic complications in Covid-19 patients. The frequency of anti-PF4/heparin IgG detectable by ELISA was $8.6 \%$. This number was even lower than that observed in a prospective study in non-Covid-19 intensive care unit patients, in whom we found 17.2\% anti-PF4/heparin IgG detected by ELISA and 5.5\% testing positive by platelet activation test 10 days after admission without signs of heparin-induced thrombocytopenia. ${ }^{32}$ None of the Covid-19 patients showed heparin-dependent plateletactivating antibodies, while the frequency of PF4-dependent platelet-activating antibodies was only $1.9 \%$ (4/222). Moreover, the reactivities of the 4 Covid-19 patient sera were all weak versus the generally strong reactivities seen with VITT sera (lag times, median 15 minutes versus < 2-5 minutes, respectively). 
Overall, Covid-19 patients with anti-PF4/heparin antibodies and PF4-dependent platelet-activating properties showed clinical characteristics similar to that of the Covid-19 patients without antiPF4/heparin antibodies; in particular, none of them developed thrombosis. In our patient cohort, thromboembolic events occurred in $4.5 \%$ of patients, with no CVST detected. Only one patient with thrombosis was reactive in the PF4/heparin ELISA but that patient's serum did not activate platelets, either in the presence of heparin or PF4. This indicates that thrombotic events in Covid-19 patients are not associated with the presence of the same anti-PF4 platelet-activating antibodies identified in vaccinated people who develop VITT.

Patients with Covid-19 and most individuals after SARS-CoV-2 vaccination express antibodies against the spike protein. ${ }^{33-35}$ However, the affinity-purified anti-PF4 and anti-PF4/heparin antibodies from sera of VITT patients did not bind to full-length spike protein, the S1 domain, or the RBD domain, but strongly bound in the PF4/heparin ELISA, and induced strong PF4-dependent platelet activation. In contrast, tested sera from Covid-19 patients strongly bound to the spike protein (Figure 2). This further indicates that the immune responses to both proteins are independent of each other.

Taken together, our findings make it unlikely that cross-reacting antibodies recognizing similar antigenic epitopes on SARS-CoV-2 spike protein and PF4 induced by vaccination are the reason for the severe thrombotic complications post-vaccination with COVID-19 Vaccine AstraZeneca. Our results also make it unlikely that anti-SARS-CoV-2 spike protein antibodies are responsible for thrombotic complications in most Covid-19 patients. This information is critical for further risk-benefit assessment of the ongoing large vaccination programs as our findings make it unlikely that the intended immune response against the SARS-CoV-2 spike protein itself induces severe VITT. Elucidating the underlying mechanism by which vaccination against SARS-CoV-2 spike protein rarely induces anti-PF4 antibodies causing VITT is urgently warranted. However, our study indicates there is no apparent need to change the SARS-CoV-2 antigen target for the vaccination strategy.

\section{Declarations}

All COI information is given in the Supplementary Files.

\section{Author contributions:}

AG, KS and TT developed the concept of the study; AA and SR produced the recombinant spike protein constructs; JM, MM, JCH, and OTK developed the COVID-19 Registry of the LMU Klinikum (CORKUM) and organized sample and data transfer; DD, AL, SR collected reported cases from cohort Freiburg and organized sample and data transfer; MPG, KALM collected reported cases from cohort Tuebingen and organized sample and data transfer; $\mathrm{CS}, \mathrm{MN}$ and $\mathrm{KH}$ collected reported cases from cohort Greifswald and organized sample and data transfer; $G L, A V, A F, P L$, and $A S$ collected reported cases from cohort Brescia and organized sample and data transfer; RM provided the structural analysis and comparison of the PF4 and spike protein; RP and JW performed the IgG affinity purification, in vitro antibody cross-reactivity 
experiments, analyzed the data, and prepared the figure; AG, KS, and TEW analysed the data; AG, KS, JM, TEW, TT wrote the manuscript. All authors have critically revised and approved the final version of the manuscript.

\section{Funding}

Sources of Funding (Tuebingen): This work was supported by the German Research Foundation (DFG) Project number 374031971-TRR 240 and by the Ministry of Science, Research and the Arts of the State of Baden-Württemberg (COVID-19 Funding). The funder had no role in study design, data collection, data analysis, data interpretation, or writing of the manuscript.

Sources of Funding (Munich CORKUM is funded, in part, by the Federal Ministry of Education and Research (BMBF) initiative "NaFoUniMedCovid19" (01KX2021), LMU excellent, the Free State of Bavaria under the Excellence Strategy of the Federal Government and the States, and the Faculty of Medicine of the LMU München. The funder had no role in study design, data collection, data analysis, data interpretation, or writing of the manuscript.

Sources of Funding (Freiburg): This work was supported by the German Center for Infection Research and the Federal Ministry of Education and Research, Germany (grant number 8039801926).

Sources of funding (Greifswald): This work was supported by the Deutsche Forschungsgemeinschaft (DFG) project number 374031971-TRR 240. G.L. is supported by a "Gerhard Domagk" research grant awarded by the University of Greifswald.

Sources of Funding (Freiburg): This work was supported by the German Center for Infection Research and the Federal Ministry of Education and Research, Germany (grant number 8039801926). The funder had no role in study design, data collection, data analysis, data interpretation, or writing of the manuscript.

Sources of Funding (FLI): This work was supported by the European Virus Archive GLOBAL (EVAGLOBAL) project that has received funding from the European Union's Horizon 2020 research and innovation program under grant agreement No 871029". The funder had no role in study design, data collection, data analysis, data interpretation, or writing of the manuscript.

Sources of funding (Frankfurt/ Main): This work is funded by DFG grants Ma 1876/12-1 and Ma 1876/13-1, grant 2018.070.1 and 2018.070.2 from the Wilhelm Sander foundation, and supported by the Corona-Funding Fonds of the Goethe-University to RM.

\section{Acknowledgement}

We thank Sarah Gekeler (University Tuebingen) and Theresa Winter (University Greifswald) and their teams for excellent logistic and technical support of biobank facilities; Manuela Gerber (University Medicine Greifswald) for patient recruitment. 
We greatly appreciate sample management and laboratory support of the technologists Ulrike Strobel, Carmen Freyer, Ricarda Raschke, Ines Warnig and Nicole Lembke. We thank Antje Westphal for the support in data collection and result documentation.

We would like to thank all local investigators, staff and especially our patients and their families for their participation in the 5 independent patients' registries.

\section{References}

1. Huang Y, Yang C, Xu XF, Xu W, Liu SW. Structural and functional properties of SARS-CoV-2 spike protein: potential antivirus drug development for COVID-19 Acta Pharmacol Sin 2020;41(9):11411149.

2. Hoffmann M, Kleine-Weber H, Schroeder S, et al. SARS-CoV-2 Cell Entry Depends on ACE2 and TMPRSS2 and Is Blocked by a Clinically Proven Protease Inhibitor Cell 2020;181(2):271-280 e8.

3. Cavaleri M, Enzmann H, Straus S, Cooke E. The European Medicines Agency's EU conditional marketing authorisations for COVID-19 vaccines Lancet 2021;397(10272):355-357.

4. W. H. O. Ad Hoc Expert Group on the Next Steps for Covid-19 Vaccine Evaluation. A minimal common outcome measure set for COVID-19 clinical research Lancet Infect Dis 2020;20(8):e192-e197.

5. Human regulatory: COVID-19 vaccines: authorised, European Medicines Agency, (https://www.ema.europa.eu/en/human-regulatory/overview/public-health-threats/coronavirusdisease-covid-19/treatments-vaccines/vaccines-covid-19/covid-19-vaccines-authorised\#authorisedcovid-19-vaccines-section) accessed 03/30/2021.

6. Dakay K, Cooper J, Bloomfield J, et al. Cerebral Venous Sinus Thrombosis in COVID-19 Infection: A Case Series and Review of The Literature J Stroke Cerebrovasc Dis 2021;30(1):105434.

7. Mowla A, Shakibajahromi B, Shahjouei S, et al. Cerebral venous sinus thrombosis associated with SARS-CoV-2; a multinational case series J Neurol Sci 2020;419:117183.

8. Ostovan VR, Foroughi R, Rostami M, et al. Cerebral venous sinus thrombosis associated with COVID19: a case series and literature review J Neurol 2021:1-12.

9. Greinacher A, Thiele T, Warkentin TE, Weisser K, Kyrle PA, Eichinger S. A Prothrombotic Thrombocytopenic Disorder Resembling Heparin-Induced Thrombocytopenia Following Coronavirus19 Vaccination. published by Research Square. 28.3.2021 (Epub ahead of print) (10.21203/rs.3.rs362354/v1).

10. Greinacher A. Heparin-Induced Thrombocytopenia N Engl J Med 2015;373(19):1883-4.

11. Greinacher A, Selleng K, Warkentin TE. Autoimmune heparin-induced thrombocytopenia J Thromb Haemost 2017;15(11):2099-2114.

12. Nazy I, Jevtic SD, Moore JC, et al. Platelet-activating immune complexes identified in critically ill COVID-19 patients suspected of heparin-induced thrombocytopenia J Thromb Haemost 2021 (Epub ahaed of print). 
13. Althaus K, Marini I, Zlamal J, et al. Antibody-induced procoagulant platelets in severe COVID-19 infection Blood 2021;137(8):1061-1071.

14. Brodard J, Kremer Hovinga JA, Fontana P, Studt JD, Gruel Y, Greinacher A. COVID-19 patients often show high-titer non-platelet-activating anti-PF4/heparin IgG antibodies J Thromb Haemost 2021 (Epub ahaed of print).

15. ENSEMBL gene data base (ENSG00000163737).

(https://www.ensembl.org/Homo_sapiens/Gene/Summary?

$\mathrm{db}=$ core; $g=$ ENSG00000163737; $\mathrm{r}=4: 73980811-73982027 ; t=E N S T 00000296029)$ accessed $03 / 27 / 2021$.

16. National Center for Biotechnology Information (NCBI): Gene Database. (https://www.ncbi.nlm.nih.gov/gene/43740568) accessed 03/27/2021.

17. Tools: Predicting Antigenic Peptides, Universidad Complutense Madrid: Immunomedicine Group, (http://imed.med.ucm.es/Tools/antigenic.pl) accessed 03/27/2021.

18. Bioinformatics ESlo. Expasy - SIM Alignment Tool for protein sequences Expasy/ Swiss Institute of Bioinformatics, (https://web.expasy.org/sim/) accessed 03/23/2021.

19. PyMol 2.4, Schrödinger Inc., 25th January 2021 (https://pymol.org/2/) accessed 03/27/2021.

20. Berman HM, Westbrook J, Feng Z, et al. The Protein Data Bank Nucleic Acids Research 2000;28:235242.

21. Wernike K, Aebischer A, Michelitsch A, et al. Multi-species ELISA for the detection of antibodies against SARS-CoV-2 in animals Transbound Emerg Dis 2020.

22. Juhl D, Eichler P, Lubenow N, Strobel U, Wessel A, Greinacher A. Incidence and clinical significance of anti-PF4/heparin antibodies of the $\operatorname{lgG}$, IgM, and IgA class in 755 consecutive patient samples referred for diagnostic testing for heparin-induced thrombocytopenia Eur $\mathrm{J}$ Haematol 2006;76(5):420-6.

23. Eichler P, Budde U, Haas S, et al. First workshop for detection of heparin-induced antibodies: validation of the heparin-induced platelet-activation test (HIPA) in comparison with a PF4/heparin ELISA Thromb Haemost 1999;81(4):625-9.

24. Eekels JJM, Althaus K, Bakchoul T, et al. An international external quality assessment for laboratory diagnosis of heparin-induced thrombocytopenia J Thromb Haemost 2019;17(3):525-531.

25. Cai Z, Yarovoi SV, Zhu Z, et al. Atomic description of the immune complex involved in heparininduced thrombocytopenia Nat Commun 2015;6:8277.

26. Ramasamy MN, Minassian AM, Ewer KJ, et al. Safety and immunogenicity of ChAdOx1 nCoV-19 vaccine administered in a prime-boost regimen in young and old adults (COV002): a single-blind, randomised, controlled, phase 2/3 trial Lancet 2021;396(10267):1979-1993.

27. Warkentin TE, Sheppard JA, Moore JC, Cook RJ, Kelton JG. Studies of the immune response in heparin-induced thrombocytopenia Blood 2009;113(20):4963-9. 
28. Greinacher A, Kohlmann T, Strobel U, Sheppard JA, Warkentin TE. The temporal profile of the antiPF4/heparin immune response Blood 2009;113(20):4970-6.

29. Krauel K, Weber C, Brandt S, et al. Platelet factor 4 binding to lipid A of Gram-negative bacteria exposes PF4/heparin-like epitopes Blood 2012;120(16):3345-52.

30. Krauel K, Potschke C, Weber C, et al. Platelet factor 4 binds to bacteria, [corrected] inducing antibodies cross-reacting with the major antigen in heparin-induced thrombocytopenia Blood 2011;117(4):1370-8.

31. Greinacher A, Holtfreter B, Krauel K, et al. Association of natural anti-platelet factor 4/heparin antibodies with periodontal disease Blood 2011;118(5):1395-401.

32. Selleng S, Selleng K, Friesecke S, et al. Prevalence and clinical implications of anti-PF4/heparin antibodies in intensive care patients: a prospective observational study J Thromb Thrombolysis 2015;39(1):60-7.

33. Murchu OE, Byrne P, Walsh KA, et al. Immune response following infection with SARS-CoV-2 and other coronaviruses: A rapid review Rev Med Virol 2021;31(2):e2162.

34. Liu L, Wang P, Nair MS, et al. Potent neutralizing antibodies against multiple epitopes on SARS-CoV-2 spike Nature 2020;584(7821):450-456.

35. Xiang F, Wang X, He X, et al. Antibody Detection and Dynamic Characteristics in Patients With Coronavirus Disease 2019 Clin Infect Dis 2020;71(8):1930-1934.

\section{Table}

Table 1: Patient characteristics, data collected from 5 university hospitals in Germany: Freiburg $(n=42)$, Munich ( $n=55)$, Tuebingen ( $n=32)$, Greifswald $(n=32)$, Bari $(n=61)$ 


\begin{tabular}{|c|c|c|}
\hline & $\begin{array}{l}\text { COVID-19 patients without } \\
\text { thrombosis }\end{array}$ & $\begin{array}{l}\text { COVID-19 patients with } \\
\text { thrombosis* }\end{array}$ \\
\hline Number of patients, $n=222(\%)$ & $212(100)$ & $10(100)$ \\
\hline - $\quad$ female, $n(\%)$ & $93 \quad(43.8)$ & $4(40.0)$ \\
\hline - $\quad$ male, $\mathrm{n}(\%)$ & $119(56.2)$ & $6(60.0)$ \\
\hline age, median (range) & $55(0.4-88)$ & $55(23-84)$ \\
\hline$<60, \mathrm{n}(\%)$ & $146(68.9)$ & $6 \quad(60.0)$ \\
\hline$\geq 60, \mathrm{n}(\%)$ & $66 \quad(31.1)$ & $4 \quad(40.0)$ \\
\hline Outpatient care, n (\%) & $61 \quad(28.8)$ & 0 \\
\hline hospitalization, n (\%) & $151(71.2)$ & $10(100)$ \\
\hline $\begin{array}{l}\text { - General ward (\% of all } \\
\text { patients) }\end{array}$ & $122(57.5)$ & $8(80.0)$ \\
\hline $\begin{array}{l}\text { - Intensive care unit (\% of all } \\
\text { patients) }\end{array}$ & $29 \quad(13.7)$ & $2(20.0)$ \\
\hline \multicolumn{3}{|l|}{ WHO COVID-19 Score, n (\%) } \\
\hline $1-3$ & $87 \quad(41.0)$ & $3(30.0)$ \\
\hline 4-5 & $105(49.5)$ & $5(50.0)$ \\
\hline$-\quad 6-9$ & $18 \quad(8.5)$ & $2(20.0)$ \\
\hline 10 & $2 \quad(0.95)$ & 0 \\
\hline \multicolumn{3}{|l|}{$\begin{array}{l}\text { Interval from symptoms to blood } \\
\text { drawing, } \mathrm{n}(\%)\end{array}$} \\
\hline - $\quad$ Day 0-10 & $115(54.2)$ & $4(40.0)$ \\
\hline - $\quad$ Day $11-20$ & $55(25.9)$ & $5(50.0)$ \\
\hline - $\quad$ Day $21-50$ & $37(17.5)$ & $1(10.0)$ \\
\hline - $\quad>50$ days & $5 \quad(2.4)$ & \\
\hline \multicolumn{3}{|l|}{$\begin{array}{l}\text { Platelets at time of blood drawing, } \\
\text { Gpt/L }\end{array}$} \\
\hline - $\quad$ mean (range) & $239(24-769)$ & $223(82-364)$ \\
\hline \multicolumn{3}{|l|}{ PF4/heparin ELISA, n (\%) } \\
\hline$-\quad O D<0.5$ & $194(91.5)$ & $9(90.0)$ \\
\hline - $\quad O D \geq 0.5<1.0$ & $13 \quad(6.1)$ & 0 \\
\hline$-\quad O D \geq 1.0$ & $5 \quad(2.4)$ & $1(10.0)$ \\
\hline
\end{tabular}


HIPA (PF4/heparin ELISA OD $\geq 0.5)$, n

$(\%)$

$\begin{array}{lllll}- & \text { Negative } & 18 & (8.5) & 10 \\ - & \text { Positive } & 0 & & 0\end{array}$

PIPA (PF4/heparin ELISA OD $\geq 0.5), n$

$(\%)$

\begin{tabular}{|c|c|c|}
\hline Negative & $14(6.6)$ & 10 \\
\hline Positive & $4 \quad(1.9)$ & 0 \\
\hline
\end{tabular}

Outcome, $\mathrm{n}(\%)$

$\begin{array}{lll}- & \text { Survived } & 206(97.2) \\ \end{array}$

- $\quad$ in hospital deaths $\quad 6 \quad(2.8) \quad 0$

*thrombosis localization: $6 x$ LAE; $1 x$ stroke; $2 x$ portal vein, $1 x$ unknown

Figures 


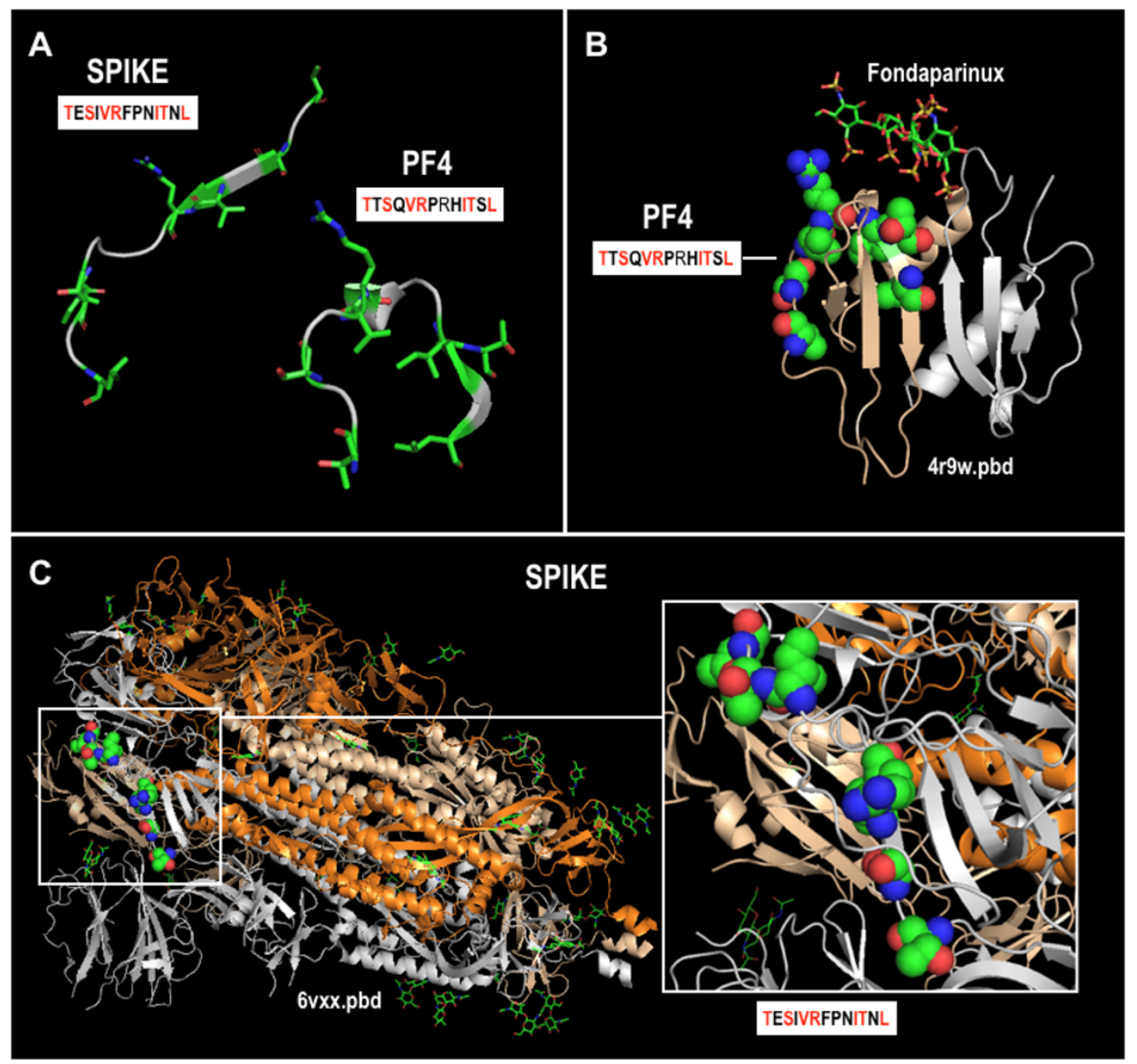

Figure 1

Comparison of the 3D-structures of PF4 and SARS-CoV-2 Spike A. The identified epitopes TESTVRFPNITNL (Spike) and TTSQVRPRHITSL (PF4 are shown in their secondary structure. Both identified linear epitopes share a ß-sheet-flexible loop structure that could initiate an unintended crossreactivity of antibodies. B. The structure of dimer PF4 with bound fondaparinux is shown. The identified epitope makes part of the binding pocket for fondaparinux. Both identified linear epitopes share a ß-sheet flexible loop structure that could initiate an unintended cross-reactivity of antibodies. C. The homologous epitope (colored spheres) is shown for one (grey structure) of the three subunits of the spike trimer which are displayed on the left. The same epitope is highlighted in the magnified inset on the right. 
A

SARS-CoV-2 S1 domain

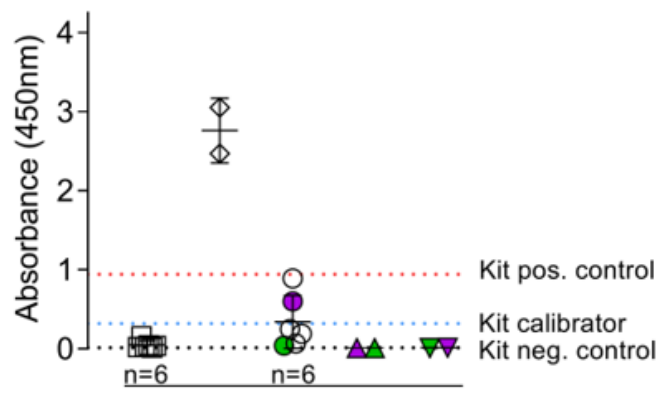

D

PF4

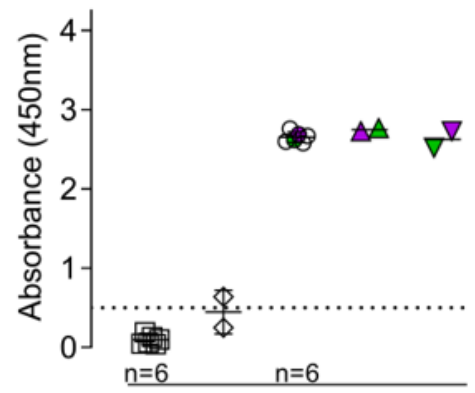

B

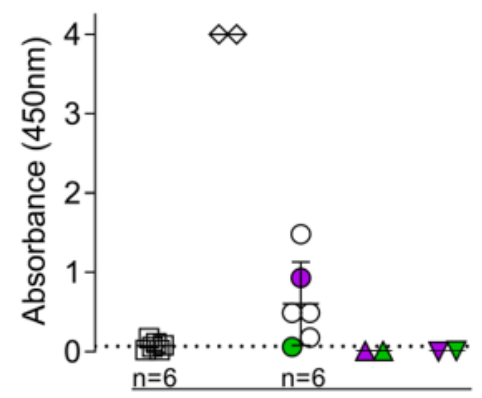

E

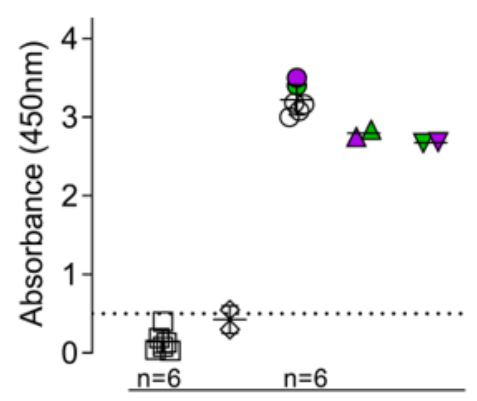

C

SARS-CoV-2 Spike full-length ectodomain

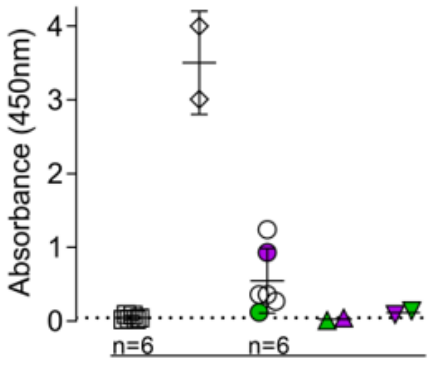

Figure legend

Serum Healthy Controls

$\diamond$ Serum Covid-19 Patients

O Serum VITT Patients

$\triangle$ PF4 Affinity Purified IgG

$\nabla \quad$ PF4/Heparin Affinity Purified IgG

\section{Figure 2}

anti-SARS-CoV-2 IgG, anti-PF IgG, and PF4/Heparin IgG antibody detection assays. Shown are the mean \pm SD of end-point enzyme-linked immunosorbent assay (ELISA) for the detection of anti-SARS-CoV-2 IgG against SARS-CoV-2 S1 domain (Panel A), RBD-SD1 domain (Panel B), Spike full-length ectodomain (Panel C), anti-PF4 IgG (Panel D) and anti-PF4/Heparin IgG (Panel E). In Panel A, dotted horizontal lines denote end-point absorbance values of anti-SARS-CoV-2 IgG against SARS-CoV-2 S1 domain assay negative control, positive control, and calibrator as provided by the assay manufacturer. In Panel $B$ and Panel $\mathrm{C}$, the dotted horizontal line denotes the median end-point absorbance value of SARS-CoV-2 negative serum specimens $(n=6)$. In Panel $D$ and Panel $E$, the dotted horizontal line represents the cut-off end-point absorbance value $(0.5$ at $450 \mathrm{~nm})$. The two VITT serum specimens across different ELISAs (denoted by green and magenta colored symbols) were used for isolation of anti-PF4 and antiPF4/Heparin IgG by affinity purification.

\section{Supplementary Files}

This is a list of supplementary files associated with this preprint. Click to download.

- COVID19ABSSupplAppendixfinal.docx

- DisclosureForms.pdf 